\title{
GENERATION Z AND THE 21st CENTURY WORKPLACE: A SCOPING REVIEW
}

\author{
Rachel A. Burger, Keiser University, West Palm Beach, Florida, U.S.A. \\ Gillian M. Fisher, Keiser University, West Palm Beach, Florida, U.S.A. \\ Allison H. Hudson, Miami Dade College, Miami, Florida, U.S.A. \\ Martha E. Rader, Keiser University, West Palm Beach, Florida, U.S.A.
}

dx.doi.org/10.18374/JABE-21-1.4

\begin{abstract}
Background: Generation Z, persons born between 1997 and 2012 and numbering over one billion, is the youngest generation to join the workforce. This has implications for employers and organizations because Generation Z (Gen Z) has preferences, values, motivations, communication styles, and expectations of work that differ from previous generations. This scoping review identifies, examines, and synthesizes the literature describing characteristics of Gen Z, factors that motivate them, their career and workplace expectations, and how employers can communicate and engage, attract and retain, and lead and manage Gen Z. In addition, literature describing the effects of the COVID-19 pandemic on Gen Z are mentioned. Methods: A methodical database search of peer-reviewed literature, dated 2012 to July 2020, was conducted using ProQuest, EBSCOHost, and Google Scholar. The review included local and international articles, but only those written in English. The reviewers examined and selected the articles that addressed Gen $Z$ in relation to the research questions that framed this scoping review. The data was charted and collated, and the results summarized and reported. The research questions include: Who is Gen Z? How can employers motivate, communicate and engage, attract and retain, and lead and manage Gen Z? What are the workplace implications of Gen Z? Results: Of the 4,683 articles identified, 348 of them met the inclusion criteria. Studies reported on the characteristics of Gen Z, Gen Z motivation, career and workplace preferences and expectations, communication and engagement, attraction and retention, and leading and managing Gen $Z$. Gaps in research were identified as well as effects of the COVID-19 pandemic on Gen Z.Conclusions: The findings indicate the impact of Gen Z on the workplace is broad and has implications for employers. The characteristics of Gen Z, their motivation, workplace preferences and expectations, communication and engagement, attraction and retention, and how to lead and manage Gen Z differ from previous generations. Employers should recognize and consider accommodating such differences. The Gen Z literature lacks research on the problems this generation faces in the workplace, their job readiness and performance, and work ethic. Also, additional research is needed to determine the impact of the use of new technologies and strategies when recruiting and retaining this generation. Future research can explore why corporate cultures that value trust and innovation, and work environments that include flexible and shared work locations and schedules, are appealing to this generation. More research is also needed to explore the short and long-term effects of adjusting management styles to suit Gen Z.
\end{abstract}

Keywords: Generation Z, Gen Z, Characteristics, Workplace, Motivation, Career, Expectations, Retention, Management, Communication, COVID-19 Pandemic 